\title{
Una didáctica interdisciplinar de la literatura infantil en inglés
}

\author{
Luis S. VILLACAÑAS DE CASTRO \\ Universitat de València \\ Departament de Didàctica de la llengua i la literatura \\ Facultat de Magisteri \\ Luis.Villacanas@uv.es
}

Recibido: julio 2012

Aceptado: febrero 2013

\section{RESUMEN}

El objetivo de este estudio es el de elaborar una propuesta didáctica que maximice el tiempo y los recursos destinados a la enseñanza del inglés durante los tres ciclos que, en España, recibe la educación primaria, con alumnos con edades comprendidas entre los 6 y los 12 años. El método escogido para lograr dicho objetivo es la interdisciplinariedad, de tal modo que pueda lograrse la síntesis de contenidos pertenecientes a distintas disciplinas y áreas curriculares, en este caso a la "Enseñanza de una lengua extranjera" (inglés) y al "Conocimiento del medio natural, social y cultural". El contexto práctico de esta propuesta, en cambio, es únicamente el conformado por las clases correspondientes a la enseñanza del inglés; y el recurso básico para lograrlo es la lectura e interpretación de textos de literatura infantil escritos en lengua inglesa. En lo que respecta a las dos áreas referidas, se toma como punto de referencia el diseño curricular activo hoy en la Comunitat Valenciana, según lo establecido en el Diario Oficial de esta comunidad autónoma (DOCV) en el año 2007.

Palabras clave: Didáctica interdisciplinar, didáctica de la lengua y literatura inglesas, didáctica de la literatura infantil, didáctica de las ciencias sociales y experimentales, educación primaria.

\section{An interdisciplinary didactics for children's literature in English}

\begin{abstract}
The aim of this study is to describe a didactic proposal to enable that teachers maximize the time and resources devoted to English teaching during the three cycles that primary education receives in Spain (students aged 6 to 12). The method selected for this purpose is interdisciplinarity, whereby a synthesis between contents belonging to different disciplines and curricular areas is reached -in this case, the contents of "Teaching and learning of English as a foreign language" and "Knowledge of the natural, social and cultural environments". However, the practical context where this proposal shall be put forward is restricted to the lessons devoted to English teaching and learning. Finally, the basic resource employed is reading and interpreting children's literature in English. As regards the aforementioned areas, the proposal uses the curricular design now functioning at the Comunitat Valenciana, which was made official by the Diario Oficial of this regional community (DOCV) in 2007.
\end{abstract}


Key words: interdisciplinary didactics, English language and literature didactics, children's literature didactics, social and experimental sciences didactics, primary education.

\section{RÉSUMÉ}

\section{L'enseignement interdisciplinaire de la littérature pour enfants} écrite en anglais

L'objectif de cette étude est d'élaborer une proposition qui maximise le temps d'enseignement et des ressources pour enseigner l'anglais en Espagne, au cours des trois cycles de l'enseignement primaire (6 à 12 ans). La méthode choisie pour atteindre cet objectif est l'interdisciplinarité, afin de parvenir à la synthèse du contenu provenant de différentes disciplines et domaines d'études, dans ce cas à «l'enseignement d'une langue étrangère» (en anglais) et «La connaissance de la naturelles, le développement social et culturel ". Le contexte pratique de cette proposition est composée uniquement de classes pour enseigner l'anglais, et la ressource essentielle est la lecture et l'interprétation des textes de la littérature pour enfants écrites en anglais. Cette étude prend comme point de départ du programme de la Comunitat Valenciana, comme indiqué dans le Journal Officiel de la région autonome (DOCV) en 2007.

Mots-clé: l'enseignement interdisciplinaire, l'enseignement de la langue et la littérature anglaise, l'enseignement de la littérature pour enfants, l'enseignement des sciences sociales et expérimentales, l'enseignement primaire.

SUMARIO: 1. Introducción, 2. Objetivos, 3. Antecedentes, 4. Metodología, 5. Resultados previstos, 6. Referencias bibliográficas.

\section{INTRODUCCIÓN}

Ya Ortega y Gasset (2010/1930) en sus escritos pedagógicos de los años 30 alertaba acerca de la desproporción existente entre los objetivos curriculares que la educación se marcaba, por una parte, y el tiempo y recursos con los que las instituciones contaban para satisfacerlos, por otra. A esta situación la llamó el problema económico de la educación (47-49). Tal desproporción no ha hecho más que incrementarse desde entonces, a medida que las ciencias han ampliado su conocimiento del mundo, y a medida también que la sociedad y su economía han intensificado sus propias demandas sobre la preparación que requieren de sus jóvenes. Una de las exigencias que más han aumentado de forma relativa respecto a las demás - lo adelantamos ya - es el conocimiento del inglés como lengua extranjera, objetivo destacado entre los propios de la etapa de primaria por el Decreto 111/2007, de 20 de julio, de la Conselleria d'Educaciò de la Comunitat Valenciana (España), en su Artículo 4 (Diario Oficial de la Comunitat Valenciana 2007: 30113). En todos los niveles educativos se percibe un creciente desequilibrio entre lo que un estudiante ha de aprender y aquello que verdaderamente aprende, pero también y sobre todo en lo que respecta al conocimiento del inglés. 
El modelo que aquí proponemos identifica la gravedad del problema y trata de ofrecerle una respuesta desde el ámbito de la didáctica de la lengua y la literatura, al que pertenece su autor. En principio, tres soluciones serían concebibles; tres serían, de hecho, las posibles maneras de enfocar el problema, y posiblemente las tres sean necesarias en cierta medida. La primera pertenecería a la esfera de la política educativa: consistiría en revisar y reducir el contenido curricular, seleccionando sólo lo más esencial para el aprendizaje. El propio Ortega y Gasset optó por esta opción, mostrando su preferencia por el conocimiento científico (2010/1930: 4950). La segunda solución correspondería al área de política económica, y tendría que ver con incrementar los recursos materiales y humanos destinados a la educación para hacer así sus objetivos más verosímiles - mas mucho nos tememos que esta segunda opción, dada la presente crisis económica, es menos viable que nunca. La tercera opción es la propiamente didáctica, que es la que aquí se presenta. El autor de este estudio pertenece al área de la didáctica, por lo que no sólo sucede que las otras soluciones no dependen directamente de él, sino que es la tercera opción la que implica la búsqueda de nuevos métodos para hacer la educación más efectiva en la consecución de sus objetivos. Dicha solución entraña la búsqueda de estrategias que permitan rentabilizar al máximo el escaso tiempo y recursos con los que actualmente cuenta la enseñanza en todos sus niveles.

Existen, empero, dificultades específicas a la "Enseñanza de una lengua extranjera" en educación primaria, un área que vamos a hacer equivalente a la asignatura de "Inglés" durante la redacción de este estudio. Tal vez sea con respecto esta área donde más se haya incrementado la exigencia institucional durante la última década, y por lo tanto (en la medida en que los recursos no se han ampliado en la misma proporción) dónde más agudo sea, también, el desequilibrio entre los objetivos y la posibilidad efectiva de cubrirlos. El Decreto 111/2007 ya mencionado refiere, en su Anexo, que «el conocimiento de lenguas extrajeras es cada vez más importante y necesario para vivir en un mundo», precisamente debido a «los progresivos avances en los campos tecnológicos e informáticos» (DOCV 2007: 30320). A esto se añade el hecho de que buena parte de la investigación y difusión científica se realiza (tanto nacional como internacionalmente) en inglés. Asimismo, la necesidad de cubrir con los niveles y objetivos marcados por el nuevo Marco Común de Referencia Europeo para las lenguas: aprendizaje, enseñanza, evaluación (Ministerio de Educación, Cultura y Deporte de España 2002) también ha elevado el baremo de exigencia por encima de las actuales posibilidades de cumplimiento, a pesar de los enormes esfuerzos realizados por parte del cuerpo docente. Existe la plena conciencia política de que espacio socio-económico internacional y competitivo requiere del aprendizaje del inglés, pero, paradójicamente, esta conciencia no se ha visto acompañada por una dotación de recursos proporcional. Todo el peso de los objetivos ha recaído, por lo tanto, sobre los maestros, que se han visto desbordados, vinculados a una causa irrealizable. 
A esta dificultad se suma otra, esta vez un problema específico de la docencia de una lengua extranjera. Consiste en su autonomía respecto a las otras áreas de aprendizaje. Ni siquiera la enseñanza de la lengua y literatura española y catalana se halla tan desvinculada del resto de materias. Ésta ha sido la causa del tradicional desprestigio que la asignatura de "inglés" ha sufrido entre el alumnado, valoración que no hacía más que reflejar el carácter externo de la asignatura dentro un plan de estudios en el cual ésta se presentaba casi como un apéndice. Tal desprestigio sólo comenzó a superarse cuando el currículum del área empezó a incorporar contenidos extra-lingüísticos, pertenecientes a otras áreas y su concreción en asignaturas. Así se refleja hoy en los Objetivos y contenidos oficiales, sobre todo en los Bloques 4 y 5: "La lengua como instrumento de aprendizaje" y "Aspectos socioculturales y consciencia intercultural", respectivamente. En dichos apartados se expresan sugerencias tendentes todas a ellas a buscar solapamientos entre el aprendizaje de la lengua extrajera y ciertos contenidos extra-lingüísticos, esto es, pertenecientes a otras áreas. Leamos, por ejemplo, el Objetivo número 6 del currículo oficial: «Usar progresivamente la lengua extranjera para afirmar y ampliar contenidos de las áreas no lingüísticas ya aprendidos y para aprender otros nuevos» (DOCV 2007: 30232). La misma idea se hace evidente en el desarrollo del Bloque 4, previsto para el 3er y último ciclo de Primaria; allí se llega al punto de animar al profesorado a realizar «tareas, pequeños proyectos e investigaciones y desarrollos de tópicos, acerca de temas relacionados con los intereses y experiencias del alumnado, o retomando, afianzando o ensanchando contenidos ya adquiridos en L1 o L2 y/o contenidos con poca demanda cognitiva de las áreas no-lingüísticas». Más aún, se propone introducir nueva información, esta vez por medio de «tareas, pequeños proyectos, investigaciones y tópicos, alrededor de contenidos sencillos de las áreas no lingüísticas no tratados todavia, complementando el currículo impartido en la L1 o L2» (30383). Estamos hablando sobre posibilidades didácticas contempladas en el actual currículum, las cuales, sin embargo, muy raramente se llevan a la práctica.

Como vemos, en todos estos casos se hace patente un intento por abrir la Enseñanza de la lengua extranjera a la realidad (social, geográfica, etc.) de los alumnos. Esta realidad, llegado el 3er ciclo de primaria, es ya bastante compleja y llena de matices. Lograr que, mediante un momento reflexivo, el estudiante adquiera conocimiento de esta realidad que lo determina y lo afecta debería ser uno de los objetivos básicos de la educación de esta etapa formativa — también para la asignatura de lengua inglesa. De hecho, tal es el enfoque cognitivo adoptado muy recientemente por Pauline Gibbons $(2009,2002)$, quien defiende que la mejor manera de enseñar y aprender una nueva lengua consiste en asimilarla a la enseñanza de contenidos específicos: «Sabemos que el uso de un nuevo lenguaje para aprender otras cosas es una forma efectiva de desarrollar una segunda lengua» (2009: 10; también 
cfr. Mohan et al. 2001). Tanto es así, que el currículum oficial del área reconoce explícitamente (en el DOCV 2007 citado) su vinculación con este enfoque, en su variante comunicativa. ${ }^{1}$ A pesar de todas estas sugerencias e indicios, lo cierto es que los contenidos extra-lingüísticos no han recibido la misma atención que los propiamente lingüísticos, ni en el currículum ni en la práctica de la enseñanza. Como resultado, las posibilidades didácticas inherentes a la integración de contenidos extra-lingüísticos procedentes de otras asignaturas se hallan también, en el presente, desaprovechadas. Contaminada tal vez por el extremo formalismo de ciertas aproximaciones tradicionales del ámbito de la lingüística (Sklovski 1970, Jakobson 1971), la enseñanza de lenguas parece estar muy distanciada de la realidad educativa y social de los docentes y alumnos, así como del más importante de sus objetivos generales: el del aprendizaje de contenidos necesarios para el funcionamiento (o la transformación) de la sociedad. No nos cansaremos de subrayar que una lengua (y todavía más todavía su aprendizaje) es indisociable de los contenidos e ideas que transmite: «Lenguaje y contenido están entrelazados de forma inextricable» (Gibbons 2009: 45). Con todo, a este aspecto la asignatura de inglés parece haberle dado la espalda, pues el aprendizaje de un segundo idioma sigue tratándose en buena medida como una tarea meramente instrumental. En otras palabras: la finalidad de que el alumno aprenda este segundo idioma no se ve acompañado de una reflexión creativa, profunda y sistemática en torno a las ideas y contenidos con ayuda de los cuales todo idioma necesariamente ha de ser aprendido; ni mucho menos en torno a una redefinición didáctica de cuáles son los contenidos extralingüísticos cuyo refuerzo y aprendizaje debería aparecer, también, como uno de los fines de la asignatura. El currículum ofrece a este respecto un amplio margen de libertad, pero la mayoría de los casos tal margen no se ha concretado en nada.

\section{OBJETIVOS}

Tal es el contexto, sin duda problemático, en el que la interdisciplinariedad se presenta como una respuesta razonable y adecuada. Antes de exponer el grado concreto en que daría solución a estos problemas, introduzcamos primero el significado concreto de esta propuesta. Siguiendo el esquema pedagógico habitual — casi el mismo que el mencionado Decreto 111/2007 utiliza-, diremos que todo currícu-

${ }^{1}$ El DOCV 2007 resume la premisa del método comunicativo de la siguiente manera: «"una lengua se aprende mejor cuando se usa para aprender", es decir, para adquirir información y conocimientos relevantes, especialmente, aquellos relacionados con los contenidos de las diversas disciplinas académicas. Prácticamente desde el principio se utilizarán conocimientos ya aprendidos en las diferentes áreas no lingüísticas, como escenario o contexto para la adquisición de la lengua extranjera, hasta que en el tercer ciclo pueda plantearse ya el afianzamiento, ampliación y adquisición de algunos contenidos nuevos, fácilmente localizables en un contexto, correspondientes a diferentes áreas no lingüísticas» (30321). 
lum consta de (1) unos objetivos, los cuales se relacionan con aquellos contenidos que la sociedad en su conjunto ha juzgado apropiado enseñar - no entraremos aquí a analizar los procesos mediante los cuales esta decisión se toma, ni la conveniencia o inconveniencia de su resultado-; (2) de una metodología, (3) de una secuenciación, y de (4) unos criterios y procedimientos para evaluar. A partir de este esquema, definimos el enfoque interdisciplinar como aquél cuya metodología, secuenciación y evaluación estarían diseñados para el objetivo de enseñar y aprender contenidos correspondientes a las asignaturas de varias áreas, tal y como estas últimas se concretan en el currículum oficial en cada nivel de formación pertinente. Desde el punto de vista de la lógica del tiempo y de los recursos ya mencionada, el enfoque interdisciplinar se presentaría como un intento por enseñar contenidos de dos o más áreas en un mismo contexto educativo espacio-temporal, y con los recursos que normalmente se emplean para abordar los contenidos de una sola.

Dicho esto, el presente estudio se propone defender la viabilidad teórica y práctica (pues a la postre se sometería al contraste empírico con el alumnado) de un enfoque interdisciplinar que trabaje con los currículos de dos áreas de educación primaria: "Lengua extrajera" (inglés) y "Conocimiento del medio natural, social y cultural". Los Objetivos generales, Contenidos y Criterios de Evaluación de una y otra se hallan detallados entre las páginas 30283-30296 y 30320-30386 del Decreto $111 / 2007$ mencionado, respectivamente. Creemos, con todo, que dicho enfoque podría ser adoptado para la enseñanza y aprendizaje de cualquier otro idioma, con mínimos cambios.

Cabe hacer la precisión de que el área que funcionaría como el contexto activo de la interdisciplinariedad sería la "Enseñanza de la lengua extranjera", sin embargo. Desde la asignatura de inglés se reforzarían algunos de los contenidos que figuran como objetivos del área "Conocimiento del medio natural, social y cultural", pero esto no sucedería a la inversa. No se trata, así pues, de una relación recíproca y simétrica: el único contexto donde la interdisciplinaridad se ejercerá de forma activa será la docencia y enseñanza del inglés. Por así decirlo, ésta aparece como el sujeto agente de la propuesta, mientras que el área "Conocimiento del medio natural, social y cultural" hará la función de sujeto paciente de la interdisciplinaridad, aquél cuyos contenidos serán revisados en las clases de inglés. De este hecho veremos derivarse todo tipo de consecuencias teóricas y prácticas que son esenciales para todos los apartados de la propuesta: diseño de secuencias didácticas, secuenciación, métodos de evaluación, etc. En relación a todos estos elementos, el currículum y contexto práctico de la enseñanza de inglés figurarán siempre como el punto de partida.

\section{ANTECEDENTES.}

La didáctica de la lengua inglesa cuenta con una larga tradición activa en la adopción del enfoque interdisciplinar, como se apreciará en el punto sexto de este estudio, la Bibliografía. Como no podía ser de otra manera, la mayoría de estas 
propuestas provienen del marco académico del mundo anglosajón, el cual (debido a características sociológicas especiales, tales como son su altísima y continua inmigración) se ha visto obligado a prestar atención al alumno angloparlante nativo tanto como al que se halla aprendiendo inglés como segunda lengua. Pauline Gibbons demuestra que esta diferencia, al menos en ciertos contextos, no es tan evidente como pudiera parecer (2009: 8). Sin duda, uno de los enfoques que se han mostrado más propicios para la enseñanza interdisciplinar es el topic-based teaching, esto es, el de una enseñanza de la lengua orientada por un tema concreto. Según Scott \& Ytreberg (2004/1990), por ejemplo, «el topic-based teaching le permite a uno organizar su material para adecuarse mejor al contexto general de su enseñanza; trabajar a través del currículum de un modo que no lo consiente la enseñanza del idioma que se basa en la estricta estructuración de contenidos, ni aquélla basada en un libro de texto» (85). Este es el mismo enfoque que Gibbons amplía, redimensiona e integra en un proyecto pedagógico que no elude responder a las demandas que el ideal de justicia social hace a la educación, en todos sus niveles y manifestaciones. Pero antes de referirnos a esta autora, analicemos algunos ejemplos previos del aprendizaje interdisciplinar de la lengua inglesa.

Fue tan pronto como en 1966 cuando la Universidad de Oxford publicó un volumen de la serie English Studies Series que reunía «pasajes breves y nosimplificados» de libros o ensayos dedicados a temas de antropología, psicología, educación, lenguaje y filosofía. Este volumen, editado por M. J. Clarke (1970/1966), incluía fragmentos firmados por Raymond Firth, Russell, A. J. Ayer, Isaiah Berlin, Simeon Potter, junto con otros autores o investigadores preeminentes. Cada extracto se acompañaba por un glosario y una serie de ejercicios lingüísticos que tomaban dicho texto como punto de partida. Aunque el volumen se dirigiese a estudiantes cuyo nivel de inglés era intermedio-alto - y aunque algunos de los fragmentos ya debieran parecer entonces tan problemáticos como resultan hoy en día-, esta iniciativa editorial continua siendo interesante en la medida en que no sólo «trata[ba] de ayudar a los estudiantes a entender el inglés y a usarlo a la hora de expresarse en estas disciplinas especiales» (ix), sino que también pretendía inculcarles contenidos, contribuir a la cultura general del estudiante, en esta caso en el campo de las ciencias sociales. Lo hacía precisamente al ofrecerles «ejemplos y fuentes que el estudiante podrá usar en su propia escritura» (ibid.). Como recurso lingüístico, su objetivo era el de formar en el aprendizaje del inglés como lengua extranjera; pero el volumen también hacía gala de la imaginación y coraje suficientes para plantearse objetivos más altos, relacionados con la satisfacción de un proyecto educativo integral en las humanidades.

Por lo que respecta a la bibliografía contemporánea, y además pertinente para el campo de la educación primaria, destacamos que Susan Helliwell publicó en 2004 un trabajo que reunía actividades destinadas a «integrar el trabajo lingüístico con el de otras asignaturas» (2004: 130). Éste es, como hemos dicho, el enfoque metodológico que planteamos en este estudio. El libro de S. Helliwell Teaching English in 
the Primary Classroom, resume de forma muy clara la manera en la que la interdisciplinariedad se ha comprendido y puesto en práctica habitualmente - siempre que se ha dado el caso, por supuesto; pues, de hecho, son demasiadas las ocasiones en los que el maestro no logra superar la idea bastante extendida de que «la integración de contenidos es un objetivo poco realista» (2004: 131). Antes bien, lo más común es que prevalezca la idea de que «no hay tiempo para ello en un temario ya saturado» (vi), como escribe H. Svecova (2009/2003), otra autora de cuyas investigaciones también nos haremos eco; o bien que, sencillamente, el propio maestro considere que no se halla «lo suficientemente formado para realizar tareas intercurriculares» (ibid.). En franco disenso con esta opinión, Helliwell defiende la interdisciplinariedad y presenta las cuatro maneras en las que, según ella, ésta podría ser abordada: «[1] usar el trabajo realizado en la asignatura de lengua extranjera como base para el trabajo en otras clases; [2] tomar algunas de las técnicas que los niños aprenden en otras asignaturas para promover el trabajo en lengua extranjera; [3] usar temas pertenecientes a otras asignaturas dentro de la clase de lengua extranjera; [y 4] enseñar otras asignaturas completamente en el idioma-destino que se pretende enseñar» (133).

Como veremos a continuación, existen razones para defender que la cuarta propuesta - consistente en el método CLIL (Content Language Integrated Learning), AICLE en castellano: Aprendizaje Integrado de Contenidos en Lengua Extranjera - ha sido empleada recientemente en el sistema público de educación valenciano, mas con resultados poco satisfactorios, hasta el punto de que la Conselleria Valenciana ha considerado a bien retirarlo. Por su interés, analizaremos este caso práctico al final de esta misma sección. Independientemente de este tema, sin embargo, cabe destacar que el libro de Helliwell presenta muchos y muy interesantes ejemplos acerca de cómo algunas técnicas usadas originariamente para favorecer el pensamiento y el lenguaje matemáticos — por ejemplo: las gráficas de tarta o de bloques, el uso de intersecciones de conjuntos, los conectores lógicos, etc.- - pueden introducirse con éxito en las clases de lengua extranjera para fortalecer el pensamiento abstracto y los procesos mentales de categorización (Helliwell 2004: 133137). En coherencia con este planteamiento, la autora pone como ejemplo un sencillo experimento de laboratorio que diseñó para la clase de inglés de primaria, «una mini-presentación para el aula de idiomas que enlaza con el trabajo que después se llevará a cabo en la otra asignatura conducida en la lengua materna» (138).

Todavía cabe mencionar más ejemplos que evidencian que la interdisciplinariedad en el área del aprendizaje de una lengua extranjera es un método eficiente en muchos sentidos. El cuaderno Cross-curricular activities, de la mencionada Hana Svecová (2009/2003) y publicado por la universidad de Oxford, presenta nada menos que treinta talleres a través de los cuales el alumno de primaria puede familiarizarse, desde la asignatura de "Inglés", con contenidos propios de las materias de historia, biología, geografía, matemáticas, literatura, educación física e incluso música. Y lo interesante es que puede hacerlo a la vez que aprende o refuerza su 
propio conocimiento de los tiempos verbales del inglés $(6,14,20-24,30-32,48,50$, $52,56,60)$, de las preposiciones $(16,34,60)$, de sus formas interrogativas $(10,44)$, o parcelas de vocabulario específico, asociadas a cada una de estas disciplinas (28, 36, 38, 42, 44, 46, 58). Este material docente es extremadamente valioso como posible modelo del producto que nuestro proyecto se propone alcanzar en la forma de secuencias didácticas adaptadas a la realidad curricular de nuestra comunidad autónoma.

Finalmente, acabaremos este resumen sobre el estado de la cuestión remitiéndonos a un antecedente positivo y uno negativo de la interdisciplinariedad. Precisamente, del lado favorable debemos destacar la que tal vez sea la iniciativa más inspiradora de nuestra propuesta, el trabajo realizado hasta la fecha por Pauline Gibbons. La matriz de su investigación gira en torno a la premisa de que el mejor currículum es aquél cuyos contenidos y metodología proponen desafíos intelectuales a los alumnos, a la vez que les ofrece un apoyo proporcional. Promueve, pues, el enfoque cognitivo. Esta idea se ve enriquecida por la segunda tesis demostrada en sus libros - de que este tipo de currículum es precisamente el más efectivo de cara a lograr una mayor homogeneización al alza entre los estudiantes de un aula diversificada social, cultural y lingüísticamente hablando; de cara, pues, a garantizar que la educación trabaje en beneficio de la igualdad de oportunidades entre alumnos de diversa procedencia socio-económica. ${ }^{2}$ En lo que respecta a la enseñanza específica de la lengua inglesa, estas dos premisas previas desembocarían - a nuestro parecer - en que la mejor manera en la que el área podría adaptarse a dicho formato curricular, y hacerse así partícipe de sus beneficios, sería abriéndose a los contenidos de las restantes materias. Todo ello lo resume Gibbons diciendo que, «lejos de ignorar las aptitudes y los conocimientos del currículum tradicional y el aprendizaje lingüístico y literario que es esencial para alumnos de inglés, el currículum que proponga desafíos intelectuales y que se oriente a la vida real de los alumnos ofrecerá más oportunidades para el aprendizaje de la lengua (también para su enseñanza), justamente porque estas aptitudes y conocimientos se presentarán dentro en un contexto significativo. En tanto es así, podrá ponerse al servicio de metas educativas más amplias e integradas en tareas auténticas, que impliquen a los estudiantes en un trabajo colaborativo y basado en el lenguaje» (Gibbons 2009: 12).

Por otra parte, en lo que respecta a los antecedentes negativos de la interdisciplinariedad, vale la pena señalar uno cuya hipótesis inicial claramente se distancia de la acogida por esta propuesta. Para ello, haremos bien en recordar la distinción cuadripartita que S. Helliwell (2004) realiza sobre las posibles versiones de la in-

2 «Las investigaciones emprendidas por Newmann et al. (1996) han demostrado que elevar la demanda académica de todos los estudiantes lleva a que todos ellos alcancen a la postre rendimientos más altos y a que se reduzcan las desigualdades de oportunidades que existían entre ellos de inicio» (Gibbons 2009: 12). 
terdisciplinaridad. Destacamos la cuarta tentativa: «desarrollar otras asignaturas en el idioma-destino que se pretende enseñar» (133)». Esta definición puede servirnos para distinguir nuestra iniciativa del método CLIL (Muñoz 2001: 33-36) o enfoque plurilingüe (Whittaker \& Linares 2011), adoptado por la Conselleria d'Educaciò de la Comunitat Valenciana a través de la Orden de 10 de junio de 2008, y animado por la Comisión Europea en el área de las lenguas. ${ }^{3}$ Dicho enfoque lleva ya años aplicándose en varios contextos educativos, sobre todo en relación al aprendizaje de las lenguas cooficiales del estado español, en las diversas regiones que se ven enriquecidas por ellas. Su uso para la enseñanza de la lengua extranjera, sin embargo, no está tan extendido en España, al menos no en el sistema público de educación. La mencionada Conselleria d'Educaciò trató de ser pionera en este aspecto, mas creemos que en este caso valenciano se hizo con poca fortuna. Por medio del Art. 3 de la orden mencionada, se implementó la enseñanza de la asignatura "Educación para la ciudadanía y los derechos humanos" en inglés, en el segundo curso de la educación secundaria obligatoria (DOCV 2008: 66289). Desde el punto de vista didáctico, es obvio que dicho enfoque no era puramente interdisciplinar. En teoría era plurilingüe, pero sobre todo era contradictorio: por un lado, justificaba el uso del inglés como lengua vehicular de dicha asignatura aludiendo a la importancia del aprendizaje de esta lengua extranjera (ibid.); por el otro lado, sin embargo, la asignatura en modo alguno contribuía a dicho aprendizaje, pues todo su diseño curricular daba por supuesto que la lengua ya había sido aprendida, esto es, que la competencia del alumnado en lengua extranjera (y la del profesorado especializado) era ya lo suficientemente alta como para que el uso del inglés no fuese un obstáculo en relación a los objetivos normales de la asignatura. En tanto es así, como es característico del CLIL, no se destinaba una sola hora a la enseñanza específica del inglés dentro de "Educación para la ciudadanía y los derechos humanos", único factor que podría haber hecho de ella una asignatura verdaderamente interdisciplinar. Es esto, a su vez, lo que lo separa en buena medida de la propuesta de Gibbons, quien propone que «las asignaturas de contenidos no-lingüísticos tienen el potencial de convertirse en los mejores contextos para desarrollar una segunda lengua en el colegio» (Gibbons 2009: 9) siempre que, por supuesto, los profesores

${ }^{3}$ Como establece la sección de lenguas de la Comisión Europea, el enfoque CLIL «consiste en la enseñanza de una asignatura curricular en un idioma diferente al usado normalmente. La asignatura podrá ser completamente ajena al aprendizaje de la lengua, como ocurriría con una clase de historia que enseñase en inglés en un colegio de España. CLIL está implementándose y revelándose efectivo en todos los niveles de la educación, desde primaria a la educación superior o con adultos. Su éxito ha ido creciendo durante los últimos 10 años y continua haciéndolo» (http://ec.europa.eu/languages/languageteaching/content-and-language-integrated learning_en.htm.) 
de dichas asignaturas sean conscientes de ello, y «dediquen tiempo al desarrollo literario de los estudiantes» (8), algo que no se daba en el caso valenciano. Por razones como ésta, a la postre las expectativas en torno al nivel de inglés de alumnos y de profesores se revelaron completamente irreales, lo cual fue en perjuicio de las dos áreas de conocimiento implicadas: tanto de "Educación para la ciudadanía y los derechos humanos" (cuyo contenidos quedaban pendientes mientras se usase el inglés como lengua vehicular), como del propio inglés, que quedaba desprestigiado como resultado de un uso inconsecuente que iba en contra de toda razón de ser didáctica.

\section{METODOLOGÍA}

Pasemos a analizar los aspectos metodológicos esenciales de la propuesta interdisciplinar que aquí presentamos. En este punto cabe resaltar, en primer lugar y a modo de condición de posibilidad para la realización de este modelo, el que la formación que hoy recibe el futuro maestro (el estudiante universitario de "Grado de Maestro/a en Educación Primaria") sea generalista. ${ }^{4} \mathrm{Al}$ menos lo es en la Universitat de València (2009), por el plan de estudios elaborado bajo la influencia de la Declaración de Boloña (1999). En segundo lugar, y esta vez en relación a los recursos requeridos, cabe destacar que la herramienta que esta propuesta identifica como la más adecuada para lograr una didáctica interdisciplinar es la literatura infantil en lengua inglesa. La elección de este instrumento también lo particulariza del resto de aproximaciones del pasado, la mayoría de las cuales solían recurrir al texto científico como herramienta didáctica (Svecovà 2009/2003, Helliwell 2004, Gibbons 2002, 2009). Por otro lado, sabemos que los intentos por integrar la literatura en el aula de inglés en primaria (Morgan \& Rinvolucri 1984, Collie \& Slater 1988, Lazar 1993, Duff \& Maley 1996, Taylor 2000, Wajnryb 2003, etc.), excepto en contadas excepciones, lo han hecho exclusivamente en beneficio del aprendizaje de la lengua, sin añadir a ello el objetivo suplementario de tratar contenidos de otras materias. En cambio, en nuestro caso la lectura de obras literarias infantiles en inglés y por parte del alumnado será la plataforma que permita al maestro sintetizar los dos objetivos que este proyecto se asigna: la enseñanza de nuevos contenidos lingüísticos, por un lado, y el refuerzo de elementos extra-lingüísticos, pertenecientes al área de "Conocimiento del medio natural, social y cultural", por otro.

Dediquemos unas palabras al fenómeno literario y a por qué consideramos que sintoniza perfectamente con la función didáctica que aquí le estamos asignando. Sin necesidad de adentrarnos en una reflexión demasiado profunda, resulta evidente que toda obra participa tanto del lenguaje como de la realidad socio-económica, e incluso natural. Siendo así, independientemente del grado de sofisticación que

\footnotetext{
${ }^{4}$ www.uv.es/graus/social/educacioprimaria.htm.
} 
uno quiera adoptar respecto a la interpretación de fenómeno literario, la necesidad de adoptar un enfoque interdisciplinar aparece como una exigencia metodológica básica si uno aspira a comprender en él la totalidad de estas dimensiones. Un escritor se ve determinado por causas puramente lingüísticas, sin duda, pero también socio-históricas, ideológicas, psíquicas, etc. A modo de ejemplo, Pierre Macherey (1966) (quien ha ofrecido uno de los más valiosos modelos de crítica literaria contemporánea) defiende que «diversas disciplinas tales como la lingüística, la teoría del arte, la teoría de la historia, la teoría de la ideología, la teoría de las formaciones inconscientes deben colaborar en la empresa [de convertir a las obras literarias en el objeto de una ciencia específica] - pues sin ellas dicha ciencia permanecería incompleta y sería tal vez imposible» (Macherey 1966: 59). Lo mismo sucede con las aportaciones de Fredric Jameson (1981: 35-36), Josep Ballester (1998), o el propio Jack Zipes (2002/1979, 1991/1983), experto en análisis del folklore, los cuentos de hadas y la literatura infantil europea en general. El mismo criterio ha seguido este último como editor general de la monumental The Norton's Anthology of Children's Literature. The Traditions in English (Zipes et. al. 2005), y asimismo J. L. Mickenberg y P. Nel (2008) en su magnífica colección de textos, A Collection of Radical Children's Literature. En esta última, la dimensión social del autor y de los lectores potenciales se establece de forma explícita en los textos, haciendo así posible su uso educativo. Tal ha sido, del mismo modo, la perspectiva que diferentes investigadores de la Facultat de Magisteri han tratado de hacer suyas en sus trabajos sobre literatura infantil, o literatura en general (Castellano 2011, Ibarra 2007, Mínguez 2007, Oltra Albiach 2011, Martínez Aguilar \& Moron Olivares 2010, Reyes Torres 2011 y Autor 2012).

Mas insistamos en que estas ideas no nos interesan en sí mismas (no pretendemos aportar nada nuevo a la crítica literaria), sino exclusivamente por sus implicaciones didácticas, que este modelo trata de aprovechar. Pensamos que todas estas reflexiones pueden derivar en una maximización de los recursos y del tiempo del aula que conlleve un mejor y más intenso aprendizaje educativo. En este sentido, lo esencial es darse cuenta de que la naturaleza interdisciplinar del fenómeno literario (Zipes 1991/1983: 2-3) (pues sólo una combinación de varias disciplinas puede dar cuenta de él) puede traducirse en una docencia interdisciplinar del texto literario. Ésta es la idea fundamental por la que creemos que la literatura (infantil, en este caso) aporta la herramienta más afín a nuestro enfoque. Si «entre las funciones más importantes atribuidas a la literatura », escribe Ballester (2007/1999), «figura la de ser una fuente de conocimiento » (98), entonces podrá serlo de muchas cosas a la vez; en realidad podrá serlo de todas aquellas áreas de conocimiento que incluyan objetos o determinaciones que afectan la producción de un texto literario. Por eso «la educación literaria [...] es interdisciplinaria (conecta con todo tipo de artes y de lenguajes) y comprende tanto la educación formal, escolar o académica, como las actividades personales del leer» (100). De ahí también que, según J. Badia y D. Cassany (1999. cit. en Ballester 2007/1999: 100), la educación literaria pueda 
abrirse a una dimensión estética y lingüística (no olvidemos que el objetivo principal de este proyecto sigue siendo el de la enseñanza y el aprendizaje del inglés). Creemos que a todas estas dimensiones es viable añadir la cultural y científica. Pues no hay duda de que se puede conocer una sociedad a partir del texto en el que ésta se escribió, lo cual no significa - como es obvio- que la sociedad tenga que ser tal y como la obra literaria la describe. En este caso, siguiendo el método de Macherey, Jameson (e incluso Althusser 2008/1965), se intentará guiar a los estudiantes hacia la reflexión de las causas reales que hicieron que el mundo social apareciese deformado en el texto literario.

Al usar la literatura como punto de partida de un abordaje interdisciplinar también evitamos el formalismo por medio del cual la crítica literaria ha tendido a reproducir durante demasiado tiempo el mismo aislamiento por el que la enseñanza de una segunda lengua se separaba del resto de saberes, y por lo tanto de la sociedad. Podríamos hablar, pues, de un tercer problema educativo, esta vez específico de la enseñanza de la literatura. Igual que sucedió con los dos anteriores, nuestra propuesta también tratará de afrontarlo y darle una solución. Pues el acercamiento a la literatura infantil que aquí proponemos quedaría subordinado en todo momento a los objetivos propios de las dos áreas de conocimiento implicadas, de la "Enseñanza de la lengua extranjera (inglés)" y del "Conocimiento del medio natural, social y cultural". Este hecho implica el reconocimiento de que, antes de poner en práctica nuestro modelo didáctico, sería necesario pasar por varias fases previas de preparación e investigación pertinentes. Los objetivos de las dos áreas referidas se concretan en unos contenidos específicos que encontramos en las páginas del currículum oficial, incluido en el DOCV. Siendo así, durante una primera fase de trabajo sería necesario desarrollar un análisis contrastivo de los currículos del área "Enseñanza de lengua extranjera: inglés" y "Conocimiento del medio natural, social y cultural". Este análisis partiría del conocimiento de cuáles son los contenidos asignados, en ambos casos, a cada uno de los ciclos de primaria, y a partir de ahí tendrá como objeto la agrupación y organización coherente de estos contenidos desde el punto de vista de una didáctica interdisciplinar. La propia letra de los currículos ofrecería, pues, la primera variable que cabría tener en cuenta a la hora de distribuir y organizar los contenidos de las dos áreas, como el paso previo al diseño de secuencias didácticas concretas que podrían llevarse a la práctica dentro del aula.

Hecho esto, se iniciaría una segunda fase de preparación, durante la cual se trataría de encontrar ejemplos literarios útiles (didácticamente hablando) para cubrir los objetivos de las dos áreas de conocimiento implicadas. Se buscarían libros cuya lectura por parte de los alumnos, y posterior interpretación por parte del maestro, pudieran facilitar a los niños el aprendizaje tanto de los contenidos lingüísticos como de los contenidos pertenecientes a las ciencias naturales y sociales, tal y como en ambos casos los define el currículum escolar. Para la elaboración de dicho canon interdisciplinar, se nos ocurren tres factores a tener en cuenta. Además del nivel de dificultad lingüística de las obras (que debería estar adaptado al de los 
alumnos), y además de su fecha de publicación (pues parece conveniente — salvo algunas excepciones - consultar antes las nuevas publicaciones que los libros antiguos, ${ }^{5}$ sobre todo para favorecer a los profesionales que tratan de abrirse camino en este género), el criterio más relevante a la hora de elegir entre el amplísimo panorama de la literatura infantil tendría que ser la cercanía que los textos literarios demuestren respecto a los contenidos establecidos por el currículum. En el fondo o en la forma, los libros deberán revelarse como herramientas adecuadas para introducir elementos de la lengua inglesa (léxico, gramática, sintaxis, etc.) y elementos relacionados con las ciencias naturales y sociales. Creemos que tal proceso podría desarrollarse de dos maneras diferentes. Por ejemplo, allí donde el análisis contrastivo revele claros solapamientos de los contenidos curriculares de las dos áreas, estas mismas coincidencias serán el punto de partida para elegir los textos. En este caso, se buscaría la manifestación literaria que le ayude a introducir los contenidos de forma sintética. Mas también podrá suceder a la inversa, esto es, que sea el encuentro con un texto literario infantil especialmente interesante lo que retroactivamente lleve al investigador a combinar los contenidos de las dos áreas de una manera original, siempre en relación al texto literario. En tal caso, la totalidad de la secuencia didáctica será la que se adapte al libro, y no al contrario, para aprovechar así todo lo que éste tenga que ofrecer desde el punto de vista lingüístico y conceptual. Siempre que los textos guarden una relación explícita con los contenidos curriculares, parece que ambas formas de operar estarán justificadas por igual, y serán empleadas indistintamente.

Finalmente, cabe descartar una tercera fase de trabajo durante la cual se elaboraría las secuencias didácticas que posteriormente se podrían en práctica en el aula de educación primaria. Para el diseño de estas secuencias se podría hacer uso del esquema propuesto por Dolz \& Schneuwly, que se ha revelado útil y equilibrado, y que tiene la ventaja de que nada en él impide su adaptación al enfoque interdisciplinar. Según dichos autores, una secuencia didáctica consta de tres partes claramente diferenciadas: (1) de una producción inicial, realizada por los alumnos y que permitirá que el maestro obtenga una visión de los déficits que sus estudiantes

${ }^{5}$ Los nuevos textos, además, tienen una clara ventaja sobre los antiguos, sobre todo cuando se quiere llevar a cabo una didáctica interdisciplinar, como es el caso. Resulta que los nuevos títulos están adaptados a las actuales dinámicas culturales, científicas y sociales, que en la mayoría de los casos han integrado en sus historias. Aparte de este factor pedagógico, la actual industria literaria infantil (sobre todo la anglosajona) presenta una calidad altísima, como se ha podido percibir en el último congreso "The Child and the Book 2012", celebrado en la universidad de Cambridge (Reino Unido), durante los días 29 de Marzo y 1 de Abril. El autor de este artículo tuvo la posibilidad de participar, y si algo quedó claro entonces fue precisamente que la literatura infantil en inglés está adentrándose con calidad y valentía en todos los ámbitos y esferas del saber. 
muestran en relación a las respectivas áreas del currículum (Dolz \& Schneuwly 2006: 121); (2) de un número de talleres (de tres a cinco, dependiendo de la amplitud de los objetivos), los cuales en nuestro caso habrán de girar en torno a la lectura del texto literario asignado; y por último (3) de una producción final, esta vez destinada a evaluar qué conocimientos de las dos áreas han sido adquiridos por los estudiantes a través de los talleres. El diseño de la secuencia didáctica implica la parte más trabajosa e importante de este modelo; en ella confluirían y se verían reflejados los conocimientos teóricos y prácticos adquiridos en las fases previas, y la elección de los libros de literatura infantil manifestará todas sus consecuencias. Cabe destacar, al respecto de la producción inicial y de su propósito, que ésta deberá arrojar luz sobre el nivel de inglés de los alumnos, en referencia a los objetivos previstos por el currículum para ese curso. Sobre su familiaridad con los contenidos propios de la otra área, "Conocimiento del medio natural, social y cultural", se confiará en la opinión del maestro de la asignatura, de quien se requerirá cierta colaboración. Lo cierto es que existen maneras de analizar, desde la clase de inglés y por medio de ejercicios de tipo oral u escrito, el grado de familiaridad que los alumnos muestran acerca de las ideas esenciales de la naturaleza, la sociedad, etc. Tal posibilidad, que esconde un verdadero método de evaluación interdisciplinar, ha sido explorada por el autor de este estudio (Autor 2012a), y bien podría ser adoptada para el contexto de la producción inicial. Dependerá de que se cumplan las variables necesarias para ello, relacionadas sobre todo con el nivel de inglés del estudiantado. En cualquier caso, resulta necesario recordar que el objetivo prioritario del proyecto sigue siendo el aprendizaje del inglés, y que existen buenas razones para plantear que éste se verá reforzado por un enfoque interdisciplinar, por un método que sea capaz de conectar con el temario de otras asignaturas. La revisión y refuerzo de los contenidos de estas últimas aparece, pues, como un beneficio colateral y ventaja ulterior de este método didáctico.

Recordemos, para finalizar, alguno de los objetivos de las dos áreas, e incluso avancemos ya ciertas áreas de conocimiento cuyos solapamientos resulta fácil identificar, junto con algunas obras de literatura infantil que podrían ayudar a cubrirlos. Por ejemplo: el primer objetivo del área "Conocimiento del medio natural, social y cultural" se define como el de "adquirir y utilizar correctamente de forma oral y escrita el vocabulario específico del área que permita el desarrollo de la lectura comprensiva a través de textos científicos, históricos y geográficos» (DOCV 2007: 30286). El segundo, por su parte, establece la necesidad de «conocer y valorar la importante aportación de la ciencia y la investigación para mejorar la calidad de vida y bienestar de los seres humanos» (ibid.). Pues bien, a través de la lectura de textos infantiles en inglés - y ponemos algunos ejemplos, como son (para el tercer ciclo de primaria) la serie de álbumes ilustrados por D. Macaulay durante los años 70 (1973, 1974, 1975, 1977); álbumes en los que se explicaba con precisión sencilla y didáctica el proceso de construcción de una catedral, un castillo, una pirámide, una ciudad romana o una urbe moderna-, estos objetivos podrían hacerse compa- 
tibles con el sexto y el décimo del área específica de inglés, a saber: «Usar progresivamente la lengua extranjera para afirmar y ampliar contenidos de las áreas no lingüísticas ya aprendidas para aprender otros nuevos»y, por otra parte, «Utilizar indistintamente las lenguas del currículo como herramientas de información y aprendizaje, teniendo en cuenta la competencia que se tiene en cada una», respectivamente (DOCV 2007: 30323). Y lo mismo podría sugerirse, como hipótesis, del octavo $^{6}$, noveno ${ }^{7}$ y décimo ${ }^{8}$ objetivo del área "Conocimiento del medio natural, social y cultural" con relación a los objetivos ya citados de "Enseñanza de una lengua extranjera: inglés", u otros como el décimo: «Utilizar indistintamente las lenguas del currículo como herramientas de información y de aprendizaje, teniendo en cuenta la competencia que se tiene en cada una» (30323). Creemos que así podría suceder si, por ejemplo, el maestro aprovechase la larga tradición de fábulas que, desde la literatura infantil, han escogido el tema de la extinción de una especie a manos de otra, con narraciones que metaforizaban ciertos comportamientos específicos de las sociedades humanas. Destacamos, a este respecto, la interesantísima obra $B A A A$, de D. Macaulay (1985); o la más reciente The Rabbits, de J. Marsden \& S. Tuan (2000), galardonada con el premio "CBCA Picture Book of the Year" en 1999. Como fábulas que son, estos títulos implican la ventaja de que permitirían realizar una distinción y un contraste de las leyes básicas que rigen las sociedades humanas frente a las tendencias que son determinantes para la vida y desarrollo del resto de las especies. Serían útiles, por lo tanto, para introducir de forma sencilla contenidos de las ciencias sociales tanto como de la ciencia natural, aunque a veces dichos conceptos se presentasen por oposición a los hechos que relatan las fábulas, las cuales no pretenden ser representaciones objetivas de la realidad, sino desplazamientos o metáforas de ella. Finalmente, muchos libros infantiles brindarían al maestro de inglés la oportunidad de reconocer, dentro de su clase, «las diferencias y semejanzas entre grupos y valorar el enriquecimiento que supone el respeto por las diversas culturas, que integran el mundo sobre la base de unos valores y derechos universalmente compartidos» (30286), como marca el séptimo objetivo del área de ciencias sociales y naturales en primaria. Pondremos un único ejemplo:

${ }^{6}$ «Analizar algunas manifestaciones de la intervención humana en el medio, valorarla críticamente y adoptar un comportamiento en la vida cotidiana de defensa y recuperación del equilibrio ecológico» (DOCV 2007: 30287).

${ }^{7}$ «Identificar los principales elementos del entorno natural, social y cultural, analizar su organización, sus características e interacciones y progreso en el dominio de ámbitos espaciales cada vez más complejos» (DOCV 2007: 30287).

${ }^{8}$ «Reconocer en el medio natural, social y cultural, cambios y transformaciones relacionados con el paso del tiempo e indagar algunas relaciones de simultaneidad y sucesión para aplicar estos conocimientos a la comprensión de otros momentos históricos» (DOCV 2007: 30287). 
The Other Side, de J. Woodson \& E. B. Lewis (2001), libro analizado profundamente por uno de los investigadores del proyecto (A. Reyes Torres 2012). Como también parecería verosímil y fructífero el intento por representar los procesos mediante los cuales los niños han de «adquirir y desarrollar habilidades sociales que favorezcan la participación en actividades de grupo adoptando un comportamiento responsable, constructivo y solidario» (30286) a través de libros más intimistas como Aldo (Burningham 1991), Changes (Browne 1990), Angry Arthur (Oram \& Kitamura 1982), o No! (McPhail 2009).

\section{RESULTADOS PREVISTOS}

Si antes hemos hecho referencia a la iniciativa alentada por la Conselleria d'Educaciò, esto se debe a que nuestra propuesta se presenta como su inverso. En nuestro caso, la enseñanza de lengua extrajera se presta a complementar a otra área, y no al contrario. Los objetivos concretos de "Enseñanza del medio natural, social y cultural" pueden hallarse detallados en la página 30286 del Decreto citado, y su aspiración global podría definirse como la de ayudar al alumno «a comprender la realidad del mundo que nos rodea y la transformaciones a las que está sometido» (DOCV 2008: 30284). Nuestro proyecto busca contribuir a que el maestro de lengua extranjera sea capaz de hacer compatible el fin instrumental de su enseñanza (el aprendizaje de contenidos lingüísticos) con estos fines alternativos. Tal es la exigente misión para cuya realización diseñaremos estrategias de ayuda a la docencia y a la evaluación interdisciplinar que los maestros de inglés podrán emplear en el aula. No plantearíamos esta posibilidad si no creyésemos que son muchos los posibles beneficios; nuestra hipótesis consiste en que la interdisciplinariedad contribuiría a solucionar los problemas mencionados a lo largo de las últimas páginas. Recordemos que uno era de carácter general, propio de todas las áreas y de todos los niveles formativos, mientras que el otro era específico de la enseñanza del inglés. Frente a estos, creemos que la propuesta didáctica interdisciplinar no sólo daría respuesta al problema económico de la educación, logrando un mejor aprovechamiento del tiempo y de los recursos de la enseñanza del inglés para dar cabida, en ella, a una consolidación de objetivos pertenecientes a otras áreas. Además $-\mathrm{y}$ tal vez sea esto lo más relevante- la interdisciplinariedad también daría respuesta al problema específico de la enseñanza de la lengua extranjera, a su relativa autonomía y desconexión del resto del currículo. De hecho, al abrirse al resto a áreas e incorporar algunos de sus objetivos, en realidad el área de Enseñanza extranjera lo haría en beneficio propio. Se minimizaría su perfil instrumental y se acrecentaría su capacidad para colaborar en los contenidos extra-lingüísticos del currículum formativo, lo cual no puede sino repercutir favorablemente en el diseño y en desarrollo práctico de la asignatura y en el mejor conocimiento del idioma. Lo mismo sucedería con el prestigio y la visión que los alumnos tienen de la literatura (tercer problema mencionado), la cual se incorporaría a la docencia como una herramienta estratégica y articulada con el resto del currículum. Esta valoración reportaría cla- 
ros beneficios para el fomento de la lectura (Ballester 2011: 36-39), en inglés y a la postre en la lengua nativa del estudiantado.

\section{REFERENCIAS BIBLIOGRÁFICAS}

BALLESTER, Josep (2011) "Sobre l'horrible perill de la lectura i les seues conseqüències", en Josep Ballester (ed.) Sobre l'horrible perill de la lectura, València: Perifèric Edicions \& Universitat de València: pp. 13-44.

—_. (2007/1999]) L'educació literaria, València: PUV.

. (1998) "Teorías literarias y su aplicación didáctica", en A. Mendoza (coord.) Conceptos clave en didáctica de la lengua y de la literatura, Barcelona: SEDLL, Ice Universitat de Barcelona/Orsori: pp. 297-322.

BROWNE, Anthony (1990) Changes, Londres \& Boston \& Sydney \& Auckland: Walker Books.

BURNINGHAM, John (1991) Aldo, London: Random House.

CASTELLANO, Margarida (2011) "Proposta didáctica per al álbum il-lustrat Quiero pescar el sol/I Will Catch the Sun de Neftalí de León", en Neftalí de León, Quiero pescar el sol/I Will catch the Sun", trad. cast. de C. Manuel, Paiporta: Denés.

COLLIE, Joanie \& SLATER, Stephen (1988) Literature in the Language Classroom: A Resource Book of Ideas and Activities, Cambridge: CUP.

COMUNICADO DE LA CONFERENCIA DE MINISTROS EUROPEOS RESPONSABLES DE LA EDUCACIÓN SUPERIOR (1999) Declaración de Bolonia, 19 de junio de 1999.

DIARI OFICIAL DE LA COMUNITAT VALENCIANA (DOCV) (2008) Orden de 10 de junio de 2008, de la Conselleria de Educación, por la que se establecen formas de organización pedagógica para impartir la materia Educación para la ciudadanía y los derechos humanos en educación secundaria obligatoria. [2008/7272] DOCV. Num. 5782/11.06.2008: pp. 66287-66291.

- (2007) Decreto 111/2007, de 20 de julio, del Consell, por el que se establece el currículo de la Educación Primaria en la Comunitat Valenciana [2007/9730] DOCV Num. 55562/24.07.2007: pp. 30110-30401.

DOLZ, Joaquin \& SCHNEUWLY, Bernard (2006) Per a un ensenyament de l'oral: iniciació als gèneres formals a l'escola, traducción de Joan Verdegal, Barcelona: Publicacions de l'Abadia de Montserrat.

DUFF, Alan \& MALEY, Alan (1996) Literature, Oxford: OUP.

EVANS, Janet (2009) (ed.) Talking Beyond the Page: Reading and Responding to Picturebooks, Nueva York \& Londres: Routledge.

- (2004) (ed.) Literacy Moves On: Using Popular Culture, New Technologies and Critical Literacy in the Primary Classroom, Nueva York \& Londres: Routledge \& David Fulton Publishers. 
GIBBONS, Pauline (2002) Scaffolding Language, Scaffolding Learning. Teaching Second Language Learners in the Mainstream Classroom, Portsmouth: Heinemann.

- (2009) English Learners Academic Literacy and Thinking. Learning in the Challenge Zone, Portsmouth: Heinemann.

HELLIWELL, Susan (2004). Teaching English in the Primary Classroom, Essex: Longman.

HOUSE, Susan (1997) An Introduction to Teaching English to Children, London: Richmond Handbooks for Teachers.

IBARRA, Noelia (2007) "¿Literatura Infantil y Juvenil e interculturalidad? Una mirada a la LIJ contemporánea", Primeras Noticias. Revista de Literatura. monográfico Literatura e Interculturalidad, $\mathrm{N}^{\circ}$ 224: pp. 21-28.

JAKOBSON, Roman (1971) Selected Writings II. Word and Language, La Haya \& París: Mouton.

JAMESON, Fredric (1981) The Political Unconscious: Narrative as a Social Symbolic Act, Ithaca, NY: Cornell University Press.

LAZAR, Gillian (1993) Literature and language teaching, Cambridge: CUP.

MACAULAY, David (1973) Cathedral, Boston: Houghton \& Mifflin Company.

- (1974) City. A Story of Roman Planning and Construction, Boston: Houghton \& Mifflin Company.

—. (1975) Pyramid, Boston: Houghton \& Mifflin Company.

—_. (1977) Castle, Boston: Houghton \& Mifflin Company.

- (1985) BAAA, Boston: Houghton \& Mifflin Company.

MACHEREY, Pierre (2006/1966) A Theory of Literary Production, Londres: Verso.

MALEY, Alan (1997) Creating Stories with Children, Oxford: Oxford University Press.

MARSDEN, John \& TUAN, Shaun (1998) The Rabbits, Sydney: Lothian Children's Books.

MARTÍNEZ AGUILAR, Consuelo \& MORÓN OLIVARES, Eva (2010) "Dar de leer. Propuestas para la formación literaria de futuros maestros", CLIJ: Cuadernos de literatura infantil y juvenil, Año $\mathrm{n}^{\circ} 23, \mathrm{~N}^{\circ} 236$ : pp. 53-59.

MCPHAIL, David (2009) No!, Nueva York: Frances Lincoln Children's Books.

MICKENBERG, Julia. L. \& NEL, Philip (2008) Tales for Little Rebels. A Collection of Radical Children's Literature, Nueva York \& Londres: New York University Press.

MÍNGUEZ, X. (2007) "Globalisation, exclusion and multiculturalism i Catalan literature for children and young people", en " $18^{\circ}$ Conference of the International Research Society for Children Literature", Kyoto (Japón).

MINISTERIO DE EDUCACIÓN, CULTURA Y DEPORTE DE ESPAÑA (2002) Marco Común de Referencia Europeo para las lenguas: aprendizaje, enseñan$z a$, evaluación, Madrid: Secretaría General Técnica del MECD \& Grupo Anaya. 
MOHAN, Bernard \& LEUNG, Constant \& DAVINSON, Chris (2001) English as a Second Language in the Mainstream, Harlow, UK: Pearson.

MORGAN, John \& RINVOLUCRI, Mario (1984) Once upon a Time: Using Stories in the Language Classroom, Cambridge: CUP.

MUÑOZ, Carmen (2001) "CLIL-AICLE (Aprendizaje Integrado de Contenidos y Lengua Extranjera)", en David Marsh et al. (2001) CLIL/EMILE: The European Dimension. Actions, Trends and Foresight Potential, Uni-COM: pp. 33-36.

OLTRA ALBIACH, Miquel (2011) "Multiculturalidad y nuevas estructuras familiares en la literatura infantil", Núñez, P. \& Rienda, J. (coords.) La investigación en Didáctica de la Lengua y la Literatura: situación actual y perspectivas de futuro, Granada: SEDLL: pp. 1763-1780.

ORAM, Hiawyn \& KIITAMURA, Satoshi (1982) Angry Arthur, Londres: Andersen Press.

ORTEGA Y GASSET, José (2010/1930) Misión de la Universidad y otros ensayos sobre educación y pedagogía, Madrid: Alianza.

UNIVERSITAT DE VALĖNCIA (2009) Pla d'estudis de grau en mestrela en educaciò primària: http://www.uv.es/graus/social/educacioprimaria.htm

REYES TORRES, Agustín (2012) "Jacqueline Woodson's Narrative Style in The Other Side: An African American Picture Book for Children", Language Value, Vol. 4: pp. 72-84.

SCOTT, Wendy A. \& YTREBERG, Lisbeth H. (2004 [1990]) Teaching English to Children, New York: Longman.

SKLOVSKI, Viktor (1970) Formalismo y vanguardia, trad. cast. de Agustín García Tirado, Madrid: Alberto Corazón.

SVECOVÀ, Hanna (2009/2003) Cross-curricular activities, London: Oxford University Press.

TAYLOR, Eric K. (2000) Using Folktales, Cambridge: CUP.

VILLACAÑAS, Luis S. (2012) "What is children's literature a metaphor of? Setting grounds for interdisciplinary learning at School", en "The Child and the Book Conference 2012: Towards Common Ground. Philosophical Approaches to Children's Literature", Cambridge: Homerton College y Faculty of Education of Cambridge University, 30 de marzo a 1de abril de 2012.

- (2012a) "The Role of Second-language Learning in diagnosing Students' Gaps of Knowledge. Towards a symptomatic Use of Interdisciplinarity", Procedia - Social and Behavioral Sciences, No 39: en prensa.

WAJNRYB, Ruth (2003) Stories: Narrative Activities for the Language Classroom, Cambridge: CUP.

WHITTAKER, Rachel \& LINARES, Ana (2011) "Written discourse development in CLIL at secondary school". Language Teaching Research, $\mathrm{N}^{\mathrm{o}} 15$ (3): pp. 343-362.

WOODSON, Jacqueline \& LEWIS, E. B. (2001) The Other Side, Nueva York: G.P. Putnam's Sons. 
WRIGHT, Andrew \& BETTERIDGE, David \& BUBCKBY, Michael (1986/1979) Games for Language Learning. New Edition, Cambridge: Cambridge University Press.

ZIPES, Jack (2002/1979) Breaking the Magic Spell. Radical Theories of Folk and Fairy Tales. Revised and expanded edition, Lexington, Kentucky: The University Press of Kentucky.

- (1991/1983) Fairy Tales and the Art of Subversion. The classical genre for children and the process of civilization, Londres \& Nueva York: Routledge.

ZIPES, Jack et. al. (2005) (eds.). The Norton Anthology of Children's Literature. The Traditions in English, Nueva York \& Londres: Norton. 